\title{
SURVEY OF TERRESTRIAL MOLLUSCS AND PARASITIC NEMATODES AS BIO CONTROL AGENTS IN SOME EGYPTIAN GOVERNORATES
}

\author{
Karima M. Azzam and Eman A. Abd El -Hady \\ Plant Protection Research Institute, Agricultural Research Center, Dokki, Giza
}

Received: Jan. 2, 2018

Accepted: Jan. 13, 2018

\begin{abstract}
A survey of terrestrial molluscs and their parasitic nematodes was carried out during 2014- 2016 seasons in certain Governorates (Cairo, Giza, Qaluobia, Dakahlia, Menoufia, Ismailia and Arish), where these snails are commonly distributed and infesting many economically important plants. The survey revealed the existence of seven snail species and three slugs. About 45 species of different plants including wheat, clover, citrus, mango, banana, tomato, potato, and amaranths were found to be infested with these gastropods. The survey revealed also the existence of six species of snail parasitic nematodes, which are considered bio control agents against these harmful molluscs.
\end{abstract}

Key words: Molluscs, nematodes, snails, slugs, control, Phasmarhabditis.

\section{INTRODUCTION}

Gastropods are very prevalent animals in the world. (Bishara, et al., 1968, Godan, 1983 \& Barker, 2002). In Egypt, land molluscs infest medicinal and ornamental plants, vegetables, orchard and field corps, causing damages to all the parts of plants (EIOkda, 1979, 1980, 1984 and Azzam, 1995). The last authors found that land gastropods infested more than 20 species of ornamental plants, 11 species of orchards, 9 species of vegetables, 4 species of field corps and three species of medicinal plants, thus emphasizing the necessity of controlling such harmful gastropods.

A survey for nematodes with potential for bio control of the snails Cernuella virgata (Da Costa), Theba pisana (Müller), and Cochlicella acuta (Müller) and Cochlicella barbara (Linneaus) and six local nematodes isolates were tested for their ability to kill these snail species was carried out (Charwat and Davies, 1999). The entomopathogenic nematodes Stienernema feltiae and Heterorhabditis sp. and the slug parasitic nematode, Phasmarhabditis hermaphrodita
(Schneider) were tested for their ability to kill Deroceras reticulatum (Müller). The entomopathogenic nematodes didn't kill slugs but $\boldsymbol{P}$. hermaphrodita could kill them (Wilson et al., 1994).

Coupland, 1995 studied the susceptibility of helicid snails to isolates of $P$. hermaphrodita and found that Monacha cantiana (Müller), Helix aspersa Müller, Theba pisana (Müller), Cernuella virgata (Da Costa), Cochlicella acuta (Müller) and Lymnaea stagnalis L. could be killed by $P$. hermaphrodita in the laboratory (Wilson et al. 2000) tested the susceptibility of seven snail species to $P$. hermaphrodita .

Phasmarhabditis tawfiki, Azzam (2003) was first recorded and described by Azzam (2011). Infectivity with this nematode was investigated towards some terrestrial snails and slugs, aquatic snails and some insect larvae in the laboratory by Azzam and Tawfik (2003). Infectivity and persistence of Phasmarhabditis tawfiki, Azzam in different soil types were investigated under laboratory and semi field conditions by Azzam (2004). Azzam and 
Khatab (2005) investigated the capability of $P$. tawfiki to control Eobania vermiculata Müller, Theba pisana (Müller) and insect larvae of Agrotis ipsilon (Hufengel) and Spodoptera littoralis (Boisd.) under greenhouse conditions. Williams and. Rae (2015), investigated whether Achatina fulica is susceptible to the gastropod parasitic nematode, Phasmarhabditis hermaphrodita, which has been developed as a biological control agent for slugs and snails in northern Europe.

A survey of terrestrial molluscs and their parasitic nematodes was carried out during 2001 - 2004 seasons in certain Governorates. The survey revealed the existence of 16 snail species and three slugs infested about 64 species of different plants. The survey revealed also 5 species of snail parasitic nematode (Azzam, 2006).

The present work deals with screening molluscs and nematodes associated with some terrestrial snails and slugs in some Governorates as means of bio control agents against these harmful pests.

\section{MATERIALS AND METHODS}

Samples of terrestrial snails and slugs were collected form the infested plants by hand form Cairo, Giza, Qaluobia, Dakahlia, Menoufia, Ismailia and Arish.

After identification of gastropods, every species individuals were kept in a terrarium with a suitable size to there number. Suspected and moribund snails and slugs were kept individually for isolation of associated nematodes, using the technique previously described by Azzam (1998 and 2003).

Some of isolated nematodes were prepared for microscopic examination. For this purpose specimens were directly taken from the mollusc's cadavers, kiled and fixed by hot TAF ( Triethanolamine formalin , composed of $2 \mathrm{ml}$. Triethanolamine $+7 \mathrm{ml}$. formalin $+91 \mathrm{ml}$. distilled water), then mounted in lactophenol or glycerol. Nematodes were identified according to Chitwood and Chitwood (1950), Andrassy ( 1976 and 1983), Poinar (1977), Soliman (1996) and Azzam (2003).

All gastropods were identified according to Kassb and Daoud (1964), Bishara et al. (1968), Godan (1983) and Nichols and Cooke (1990), Azzam and Tawfik (2011).

\section{RESULTS AND DISCUSSION}

Collected land snail and slugs with their host plants were recorded in Table, (1). They were listed in an alphabetic arrangement, while their associated nematodes were recorded in Table, (2). The survey revealed the existence of seven snail species belonging to two families (Eobania vermiculata (Müller), Monacha obstructa (Ferussac), Monacha cartusiana (Müller) , Theba pisana (Müller), Theba spp.( Family : Helicidae . Opeas pyrgula (Schmacker and Boettger) and Opeas pumilum (Pfeiffer) (Family : Subulinidae ) and three slugs from two family ( Limax flavus $L$. and Lehmania marginata (Müller)( Family : Limacidae) and Deroceras laeve (Müller) (Family : Agriolimacidae ).

The snails were infesting about 45 species of different plants. The most abundant and important snails species were: Monacha obstucta (Ferussac) and Eobania vermiculata (Müller). The first infested 29 plant species including, Citrus, Banana, Mango, Alfalfa , Tomato, Pepper, Lettuce, Cucumber ,Mint, Pot marigold, , Dracaena, Keshda plant, Garden palm, , Camel thorn and Silver wattle. The second snail species infested 16 plant species including Citrus, Mango, Papaya, Chamomile, Cabbage, Squash, Lettuce, Celery, Asparagus, Pot Marigold, Mandalay, Wild porcelain, and Keshda plant (Table1). 
Survey of terrestrial molluscs and parasitic nematodes as bio control..........

Table (1) : Molluscs and their host plants occurred in Egypt governorates

\begin{tabular}{|c|c|c|}
\hline Scientific name of host plant & Common name & Governorates \\
\hline \multicolumn{3}{|c|}{$\begin{array}{l}\text { 1- Family : Helicidae } \\
\text { 1-1. Eobania vermiculata (Müller) }\end{array}$} \\
\hline $\begin{array}{l}\text { Amaranthus caudatus } \\
\text { Anthemis nobilus } \\
\text { Aplum graveolens } \\
\text { Asparagus spregri } \\
\text { Brassica oleracea } \\
\text { Calendula officinalis } \\
\text { Certea papaya } \\
\text { Citrus sp. } \\
\text { Chrysanthemum frutescens } \\
\text { Cucurbita pepo } \\
\text { Euophorbia pelpus } \\
\text { Lacttucu sativa } \\
\text { Lolium prene. } \\
\text { Monestra deliciosa } \\
\text { Mangifera indica } \\
\text { Amaranthus caudatus }\end{array}$ & $\begin{array}{l}\text { Amaranths } \\
\text { Chamomile } \\
\text { Celery } \\
\text { Asparagus } \\
\text { Cabbage } \\
\text { Pot Marigold } \\
\text { Papaya } \\
\text { Citrus } \\
\text { Mandeleya } \\
\text { Squash } \\
\text { Wild porcelain } \\
\text { Lettuce } \\
\text { Gazonasa } \\
\text { Keshda plant } \\
\text { Mango } \\
\text { Amaranths }\end{array}$ & $\begin{array}{l}\text { Cairo } \\
\text { Cairo, Giza \& Qaluobia } \\
\text { Giza } \\
\text { Cairo , Giza \& Qaluobia } \\
\text { Cairo, Giza \& Qaluobia } \\
\text { Cairo } \\
\text { Giza } \\
\text { Cairo, Giza \& Qaluobia } \\
\text { Cairo } \\
\text { Giza } \\
\text { Cairo, Giza \& Qaluobia } \\
\text { Giza } \\
\text { Giza } \\
\text { Giza } \\
\text { Giza \& Ismailia } \\
\text { Cairo\& Ismailia }\end{array}$ \\
\hline \multicolumn{3}{|c|}{ 1-2. Monacha obstructa (Ferussac) } \\
\hline $\begin{array}{l}\text { C. officinalis } \\
\text { Capsicum sp. } \\
\text { Citrus sp. } \\
\text { Cichorium intylous } \\
\text { Cucumis sativus } \\
\text { C.dactylon } \\
\text { Duranta repens } \\
\text { Dracaena sp. } \\
\text { L. sativa } \\
\text { Lycopersicum crispum } \\
\text { Menthe sp. } \\
\text { M.deliciosa } \\
\text { Musa sp. } \\
\text { Petroselinum crispum } \\
\text { P.zonal } \\
\text { Phonentix sp. } \\
\text { Medicago sativa } \\
\text { Alhagi maurorum } \\
\text { Acacia dealbata } \\
\text { Mangifera indica } \\
\text { Amaranthus caudatus }\end{array}$ & $\begin{array}{l}\text { Pot marigold } \\
\text { Pepper } \\
\text { Citrus } \\
\text { Chicory } \\
\text { Cucumber } \\
\text { Grass } \\
\text { Duranta } \\
\text { Dracaena } \\
\text { Lettuce } \\
\text { Tomato } \\
\text { Mint } \\
\text { Keshda plant } \\
\text { Banana } \\
\text { Parsley } \\
\text { Pelargonium } \\
\text { Garden palm } \\
\text { Alfalfa } \\
\text { Camel Thorn } \\
\text { Silver Wattle } \\
\text { Mango } \\
\text { Amaranths }\end{array}$ & $\begin{array}{l}\text { Cairo, Giza ,Menoufia\& Arish } \\
\text { Giza, } \\
\text { Giza Menoufia, Dakahlia } \\
\text { Giza } \\
\text { Giza } \\
\text { Cairo Giza, Qaluobia Menoufia } \\
\text { Cairo, Giza\& Qaluobia } \\
\text { Cairo, Giza } \\
\text { Giza Menoufia, Dakahlia } \\
\text { Giza } \\
\text { Giza } \\
\text { Giza } \\
\text { Giza } \\
\text { Giza } \\
\text { Cairo, Giza } \\
\text { Cairo, Giza } \\
\text { Menoufia, Arish\& Dakahlia } \\
\text { Arish } \\
\text { Arish } \\
\text { Giza \& Ismailia } \\
\text { Cairo\& Ismailia }\end{array}$ \\
\hline
\end{tabular}


Table (1) : Cont.

\begin{tabular}{|c|c|c|}
\hline \multicolumn{3}{|c|}{ 1-3. Monacha cartusiana (Müller) } \\
\hline B. oleracea & Cabbage & Giza \\
\hline L.sativa & Lettuce & Giza \\
\hline M. sativa & Lucerne & Giza \\
\hline Sorghum vulgare & Sweet sorghum & Giza \\
\hline Alhagi maurorum & Camel Thorn & Arish \\
\hline Medicago sativa & S Alfalfa & Arish, Dakahlia \\
\hline Acacia dealbata & Silver Wattle & Arish \\
\hline Citrus sp & Citrus & Cairo, Giza \& Dakahlia \\
\hline Mangifera indica & Mango & Giza \& Ismailia \\
\hline Amaranthus caudatus & Amaranths & Cairo\& Ismailia \\
\hline \multicolumn{3}{|c|}{ 1-4. Theba pisana (Müller) \& Theba spp. } \\
\hline A.nobilis & Chamomile & Cairo, Giza \& Qaluobia \\
\hline C. officinalis & Pot marigold & Cairo, Giza \& Qaluobia \\
\hline \multicolumn{3}{|c|}{$\begin{array}{l}\text { 2- Family : Subulinidae } \\
\text { Opeas pyrqula (Schmacker and Boettger) Opeas pumilum (Pfeiffer) }\end{array}$} \\
\hline Chrysanthemum frutescens & Margunerite daisy & Cairo \& Giza \\
\hline Chrysanthemum sp. & Zonal geranium & Cairo \& Giza \\
\hline Pelargonium zonal & Horseshoe geranium & Cairo \& Giza \\
\hline Pothos aurus & Golden pothos & Cairo \& Giza \\
\hline Sterlizia reginae & Crane flower & Cairo \& Giza \\
\hline Tegates erecta & Marigold & Cairo \& Giza \\
\hline Tradescanthia fluminensis & Wandering jew & Cairo \& Giza \\
\hline Winka rosa & Rose & Cairo \& Giza \\
\hline Zinnia elegans & Zinnia & Cairo \& Giza \\
\hline Asparagus spregri prene & Marigold & Cairo \& Giza \\
\hline Monestra deliciosa & Keshda plant & Cairo \& Giza \\
\hline Euophorbia pelpus & Wild porcelain & Cairo \& Giza \\
\hline Petunia hybrida & Petunia & Cairo \& Giza \\
\hline Rosa sp. & Wild rose & Cairo \& Giza \\
\hline \multicolumn{3}{|c|}{ 3- Family : Limacidae Slugs: Limax flavus $L . \quad$ Lehmania marginata (Müller) } \\
\hline A. spengeri & Asparagus & Cairo \\
\hline Coleus blimei & Coleus & Cairo \\
\hline Chrysanthemum sp. & Chrysanthemum & Cairo \\
\hline C. frutescens & Mandeliya & Cairo \\
\hline E.pelpus & Wild porcelain & Giza \\
\hline P. zonal & Pelargonium & Cairo \& Giza \\
\hline P.hybrida & Petunia & Cairo \& Giza \\
\hline P.aureus & Pothos & Cairo \& Giza \\
\hline T. fluminensis & Reptile Jew plant & Cairo \& Giza \\
\hline \multicolumn{3}{|c|}{ 4- Family : Agriolimacidae Slug: Deroceras laeve (Müller) } \\
\hline Amaranthus caudatus & Amaranths & Cairo \& Giza \\
\hline
\end{tabular}


Table (2): Parasitic nematodes associated with gastropods in Egyptian Governorates

\begin{tabular}{|c|c|c|}
\hline Nematode species & Gastropod species & Governorates \\
\hline $\begin{array}{l}\text { 1- Phasmarhabditis } \\
\text { tawfiki, Azzam }\end{array}$ & $\begin{array}{l}\text { Eobania vermiculata (Müller) } \\
\text { Theba pisana (Müller) } \\
\text { Theba spp. } \\
\text { Limax flavus L. } \\
\text { Lehmaninia marginata (Müller) } \\
\text { Opeas pyrgula (S and B) } \\
\text { Opeas pumilum ( Pfeiffer) } \\
\text { Deroceras reticulatum (Müller) }\end{array}$ & $\begin{array}{l}\text { Cairo , Giza \& Qaluobia } \\
\text { Cairo , Giza \& Qaluobia } \\
\text { Cairo , Giza \& Qaluobia } \\
\text { Cairo , Giza \& Qaluobia } \\
\text { Cairo , Giza \& Qaluobia } \\
\text { Cairo , Giza \& Qaluobia } \\
\text { Cairo \& Giza } \\
\text { Cairo \& Giza }\end{array}$ \\
\hline $\begin{array}{l}\text { 2- Phasmarhabditis } \\
\text { hermaphrodita } \\
\text { (Schneider)* }\end{array}$ & $\begin{array}{l}\text { E. vermiculata } \\
\text { L. flavus } \\
\text { L. marginata } \\
\end{array}$ & $\begin{array}{l}\text { Cairo, Giza \& Qaluobia } \\
\text { Cairo, Giza \& Menoufia } \\
\text { Dakahlia }\end{array}$ \\
\hline 3- Rhabditis sp. & $\begin{array}{l}\text { E. vermiculata } \\
\text { Monacha obstructa (Ferussac) } \\
\text { L. flavus } \\
\text { L. marginata } \\
\text { Opeas pyrgula } \\
\text { Opeas pumilum }\end{array}$ & $\begin{array}{l}\text { Cairo \& Giza } \\
\text { Cairo, Giza,Menoufia \&Arish } \\
\text { Cairo } \\
\text { Cairo \& Giza } \\
\text { Cairo \& Giza } \\
\text { Cairo \& Giza }\end{array}$ \\
\hline 4- Cephalobus sp. & $\begin{array}{l}\text { E. vermiculata } \\
\text { Opeas pyrgula } \\
\text { Opeas pumilum }\end{array}$ & $\begin{array}{l}\text { Giza, Qaluobia, Dakahlia } \\
\text { Giza, Qaluobia, Dakahlia, } \\
\text { Menoufia \& Arish }\end{array}$ \\
\hline 5- Diploscapler sp. & $\begin{array}{l}\text { M.obstructa } \\
\text { T. pisana }\end{array}$ & $\begin{array}{l}\text { Cairo, Giza \& Menoufia } \\
\text { Cairo, Giza \& Menoufia }\end{array}$ \\
\hline 6- Agfa sp. & Monacha spp & Menoufia, Dakahlia,Cairo\& Giza \\
\hline
\end{tabular}

The survey revealed the presence of six parasitic nematodes (Table 2).

The surveyed parasitic nematodes were: Phasmarhabditis tawfiki Azzam, Phasmarhabditis hermaphrodita (Schneider), Rhabditis sp., Cephalobus sp. and Diploscapter sp. and Agfa sp. The first species was recorded and described as a new species of snail parasitic nematode in Egypt by Azzam (2003). Azzam (1998) recorded Rhabditis sp. for the first time in Egypt. All the isolated nematode species except the last one (Agfa sp.) were previously recorded in Egypt by Azzam, 2006.

P. tawfiki Azzam was isolated from E. vermiculata, T. pisana, Theba spp., Opeas pyrgula and Opeas pumilum snails and $L$. flavus $L$. , $L$. marginata and Deroceras lave slugs, while $P$. hermaphrodita was isolated from $E$. vermiculata, $L$. flavus and $L$. marginata. Rhabditis sp. was isolated from $E$. vermiculata, Monacha obstucta, Opeas pyrgula and Opeas pumilum snails and $L$. flavus, $L$. marginata slugs (Table 2). Cephalobus sp. was isolated from land snails ( $E$. vermiculata, , O. pyrgula and O. pumilum ). Meanwhile, Diploscapter sp. was isolated from $M$. obstructa and $T$. pisana and Agfa sp. was isolated from land snails only Monacha sp .

Glen and Wilson (1997) listed 8 species of snails including one aquatic snail, all of them were susceptible to infection with $P$. hermaphrodita but non of the exposed insects accepted the 
infection with it. Azzam recorded five species of snails including one aquatic, two species of slugs and three species of insects that were susceptible to infection with Rhabditis sp., Azzam and Tawfik (2003) recorded five species from each aquatic and terrestrial snails, two slugs, and three insect species were susceptible to infection with Phasmarhabditis tawfiki Azzam. Charwat and Davies (1999) recorded high mortality of Cochlicella acuta when exposed to two Cephalobid isolates. Williams and. Rae (2015) found that Deroceras panormitanum killed in less than 30 days of exposure to $P$. hermaphrodita at $\mathbf{3 0}$ and $\mathbf{1 5 0}$ nematodes per $\mathbf{c m}^{2}$ for 70 days while Achatina fulica show remarkably resistant to the nematode at both doses.

From the obtained results, it could be concluded that parasitic nematodes specially $\boldsymbol{P}$ tawfiki , $P$. hermaphrodita , Rhabditis sp. and Cephalobus sp., represent active biocontrol agents of land gastropods in Egypt and should be taken into consideration in the control of these harmful pests.

\section{Acknowledgment:}

The authors gratefully thanks Dr. Sayed Hagag Professor in Bee Department of Plant Protection Institute for his kind help in supplying the samples from Arish.

\section{REFERENCES}

Andrassy, I. (1976). Evolution as a basis for the systematization of nematodes Pitman Publishing London. San Francisco Mellbourne.288pp.

Andrassy, I. (1983). A taxonomic review of the suborder Rhabditina (Nematoda, Secernentia). Paris, France Orostom 241pp.

Azzam Karima, M. (1995). Studies on some malacophagous insects in
Egypt Ph. D. Thesis Fac. Agric. Cairo Univ. 326p.

Azzam Karima, M. (1998). First record of snail parasitic nematode Rhabditis sp. isolated Egyptian terrestrial snail and its capability to infect other pests. Egypt. J. Biol. Pest Cont. 8(1): 27-29.

Azzam Karima, M. (2003). description of Phasmarhabditis tawfiki Azzam n. sp. isolated from Egyptian terrestrial snail and slugs. Egypt. Ger. Soc. J. Zool. (24D): 79-87.

Azzam Karima, M. (2004). Effect of soil type on the capability of Phasmarhabditis tawfiki Azzam to infect snails under laboratory and outdoor conditions. Egypt. J. Biol. Pest Cont., 14(2): 387-390.

Azzam Karima, M. (2006). Survey on terrestrial gastropods, their host plants and parasitic nematodes as bio control agents. J. Egypt. Ger. Soc. Zool. Vol (49D) :49-61.

Azzam Karima, M. and M.M. Kattab (2005). Potential of the snail parasitic nematode Phasmarhabditis tawfiki Azzam to control some snails and insects under green house conditions. Bull. Fac. Agric., Cairo Univ., 56: 631640.

Azzam Karima, M. and M.F.S. Tawfik (2003). Capability of the nematode, Phasmarhabditis tawfiki Azzam to infect some snails, slugs and insect larvae in the laboratory. Egypt. Ger. Soc. J. Zool.(24D): 27-32.

Azzam Karima, M. and M.F.S. Tawfik (2011). First record of the sharp awl snail Opeas pyrgula (Schmacker and Boettger, 1891) and the dwarf awl snail, Opeas pumilum ( Pfeiffer,1840 ) in Egypt, and their response to climatic changes . Egypt. J. Biol. Pest Cont.21(2):325-330.

Barker, G.M. (2002). Molluscs as crop pests. CABI Publishing, Wallingford, UK. 468 pp. 
Bishara, S., M.S. Hassan and A.S. Kalliny (1968). Studies on some land snails injurious agriculture in U.A.R. Rev. Zool. Bot. Afr. LXXXII,(3-4) : 239-252.

Charwat, S.M. and K.A. Davies (1999). Laboratory screening of nematodes isolated from South Australia for potential as bio control agents of helicid snails. J. Invert. Pathol., 74:5561

Chiwood, B.G. and M.B. Chiwood (1950). An introduction to Nematology. Monumental Printing Company. 190 pp.

Coupland, J.B. (1995). Susceptibility of helicid snails to isolate nematode, Phasmarhabditis hermaphrodita from Southern France. J. Invert. Pathol., 66: 207-208.

Glen, D.M. and M.J. Wilson (1997). Slugparasitic nematodes as biocontrol agents for slugs . Agro- Food Industry Hi- Tech, in press.

Godan, D. (1983). Pest snails and slugs. Verlage, Berlin, 422pp.

El -Okda, M.M.K. (1979). Land snails of economic important at Alexandria region with some notes on the morphological features classification, economic damage and population on the ornamental plants. Agric. Res. Rev.,57(1) : 12-130.

El - Okda, M.M.K. (1980). Land snails of economic importance on vegetable crops at Alexandria and neighboring regions. Agric. Res. Rev., 58 (1) : 7985.

El - Okda, M.M.K. (1984). Land mollusca infestation and chemical control in El-
Ismaeiliya Governorate. Agric. Res. Rev., 62(1):87-94.

Kassab, A. and H. Daoud (1964). Notes on the biology and control of land snails of economic important in the U.A.R. Agric. Res. Rev., 42(4) :77-98.

Nichols, D. and J.A.L. Cooke (1990). The Oxford Book of invertebrates OxfordNew York, Toronto, Melbourne, Oxford University Press. 241pp.

Poinar, G.O. Jr. (1977). Chi key to the groups and genera of nematode parasites of invertebrates Commonwealth Agricultural Bureaux, England 43 pp.

Soliman, G.N. (1996). Invertebrate Zoology. The mideastern invertebrate fauna. Part I: The noncoelomates, 1st ed, the palm press, Cairo, Egypt, 351 pp.

Williams, A.J. and R. Rae (2015). Susceptibility of the Giant African snail (Achatina fulica) exposed to the gastropod parasitic nematode Phasmarhabditis hermaphrodita. Journal of Invertebrate Pathology 127, 122-126

Wilson, M.J., D.M. Glen, L.A. Hughes, J.D. Pearce and P.B. Rodgers (1994). Laboratory tests of he potential of entomopathogenic nematodes for the control of field slugs Deroceras reticulatum. Journa. Invert. Pathol., 64(3): 182-187.

Wilson, M.J., D.M. Glen, L.A. Hughes, G.M. Hamacher and D.M. Glen (2000). Effect of Phasmarhabditis hermaphrodita on non target molluscs. Pest Manag. Sci.,56(8) : 711716. 
حصر للرخويات الارضية والنيماتودا المتطقلة عليها كعوامل للمكافحة الحيوية فى بعض المض

المحافظات المصرية

كريمه محمود عزام ، إيمان عبد الرازق عبد الهادي الزبان

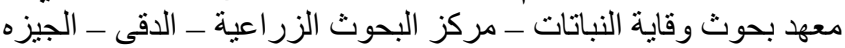

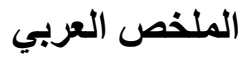

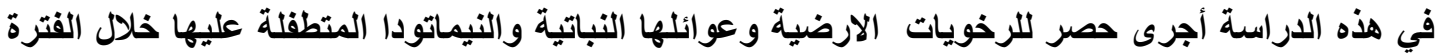

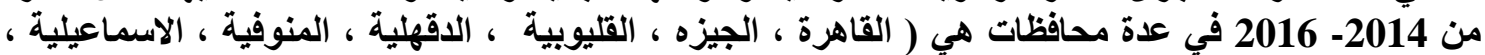

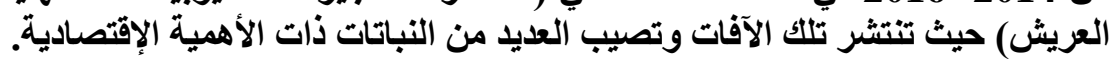

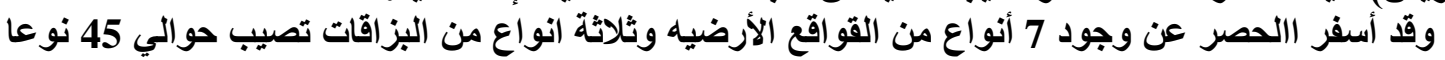

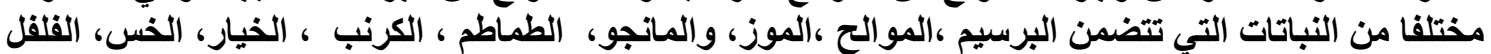

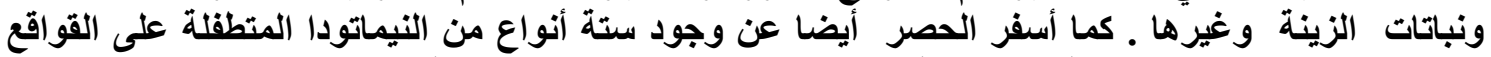
والتي تعتبر من عوامل المكافحة البيولوجية التى يمكن الإستفادة منها فى مكافحة تلك الآفات ـ 